\title{
Asymptomatic chronic hypotony due to subclinical choroidal effusion after blunt trauma
}

\author{
John Mark S. de Leon, Ronald Steven Medalle II \\ Department of Health Eye Center, East Avenue Medical Center, East Avenue, \\ Diliman, Quezon City, Philippines
}

\begin{abstract}
Objective: To present a case report of asymptomatic post-traumatic chronic hypotony in which the cause was undetected until phacoemulsification.

Methodology: Case report.

Resuts: A 55-year-old female's left eye suffered blunt trauma causing hyphema and iritis, which were successfully managed; however, up to a year after, the intraocular pressure (IOP) ranged from 3 to $5 \mathrm{mmHg}$ and the anterior chamber remained very shallow (Van Herick grade 4) with the lens-iris diaphragm pushed anteriorly with difficulty assessing the angles for recession or clefts. Visual acuity was initially 20/20 upon resolution of the hyphema but worsened to 20/40 a year after, presumably due to a developing cataract. Periodic dilated fundus examinations revealed no hypotony maculopathy or choroidal effusions. Prior to phacoemulsification, ultrasound biomicroscopy (UBM) revealed 360 degrees of mild peripheral choroidal effusions. During phacoemulsification, after intraocular lens insertion, direct gonioscopy revealed a supero-nasal cyclodialysis cleft (2 clock hours) and this was repaired intraoperatively with direct cyclopexy through a partial thickness scleral flap. Post-operatively, the vision improved to 20/20 without correction and the IOP normalized to 16 to $18 \mathrm{mmHg}$.

Conclusion: Chronic hypotony post-trauma may be asymptomatic and the cause may not be clinically evident and may be detected by UBM (choroidal effusion). In our case, the proximate aetiology (cyclodialysis cleft) of the effusion was only observed intraoperatively after phacoemulsification for which cyclopexy was performed which increased the IOP to physiologic levels.
\end{abstract}

Keywords: blunt trauma, choroidal effusion, cyclopexy, hypotony, intraocular pressure, ultrasound biomicroscopy

\section{Background}

Chronic hypotony may be due to surgery, trauma, inflammation or systemic diseases. Patient with chronic hypotony may be asymptomatic or can have blurred vision with complications. ${ }^{1}$ Since management can be either be conservative or

Correspondence: John Mark S. de Leon, Torres Bugallon Street, Tierra Pura Subd.,

Quezon City 1107, Philippines.

E-mail:jmarkmd1@yahoo.com 
aggressive, a thorough investigation of hypotony through clinical and auxiliary means is invaluable.

\section{Case presentation}

A 55-year-old female presented with blurring of vision and redness on her left eye (OS) from blunt trauma from a tossed cup. At the emergency room her visual acuity (VA) was 20/20 for both eyes; slit-lamp examination revealed traumatic hyphema, grade I, OS. Intraocular pressures (IOPs) were $10 \mathrm{mmHg}$ in both eyes (OU). Antibiotic-steroid eye drops, OS and oral tranexamic acid were prescribed. Day 3 post-trauma, the hyphema progressed to grade III with VA dropping to good light projection, necessitating admission for observation. IOP, OS ranged from 5 to $6 \mathrm{mmHg}$. The hyphema resolved on day 4 and the patient was discharged. The central anterior chamber depth, OS was shallow (Fig. 1a), in contrast to OD (Fig. 1b).

Four-mirror gonioscopy, OS showed closed angles nasally and temporally and it was difficult to appreciate any angle recession or cyclodialysis clefts even on indentation because of the lens-iris diaphragm pushed forward. IOPs, OS still ranged from 4 to $5 \mathrm{mmHg}$. A dilated retinal examination, OS revealed an unremarkable posterior pole. Upon discharge, the best-corrected VA (BCVA) was as follows:
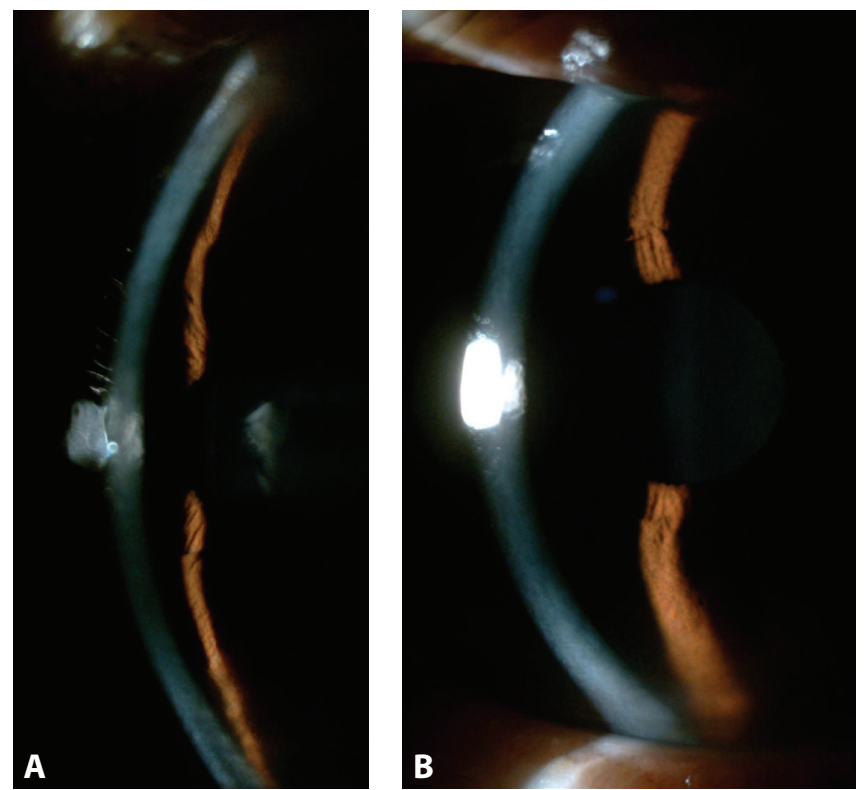

Fig. 1. (a) Central anterior chamber depth, OS. (b) Central anterior chamber depth, OD. 


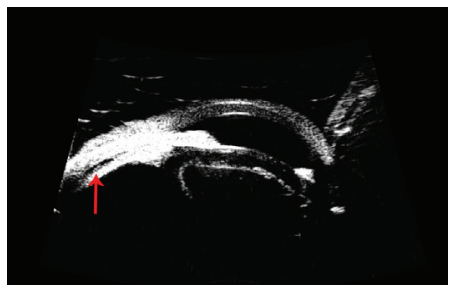

Fig. 2. UBM showing choroidal effusion, 0 S.

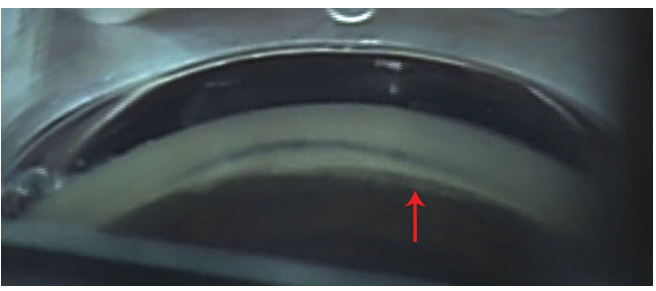

Fig. 3. Cyclodialysis cleft, OS.

OD: $+1.25-0.25 \times 90(20 / 20)$ and OS: +1.50 (20/32). On monthly follow-ups, BCVA, OS was stable at 20/32, though with progressive blurring of vision.

A year after the trauma because of the persistence of asymptomatic hypotony, an ultrasound biomicroscopy (UBM) was done, OS showing closed angles as well as 360 degrees of choroidal effusion (red arrow; Fig. 2); however, no cyclodialysis cleft was observed in any of the cross-sectional UBM cuts.

Since the cataract progressed and BCVA dropped to 20/40, phacoemulsification with intraocular lens insertion was performed. Immediately after lens insertion, the anterior chamber was filled with viscoelastic and an intraoperative gonioscopy with a direct gonioprism revealed a 2-clock hour supero-nasal cyclodialysis cleft (red arrow; Fig. 3).

The conjunctival area overlying the cleft was marked, a conjunctival peritomy and a partial thickness scleral flap was dissected anteriorly to the ciliary body. Direct cyclopexy was then completed at the site with nylon 10-0 sutures (Fig. 4).

One-week after cataract surgery and cyclopexy, OS, VA improved to 20/20, the anterior chamber was formed with open angles and the IOP was $18 \mathrm{mmHg}$.

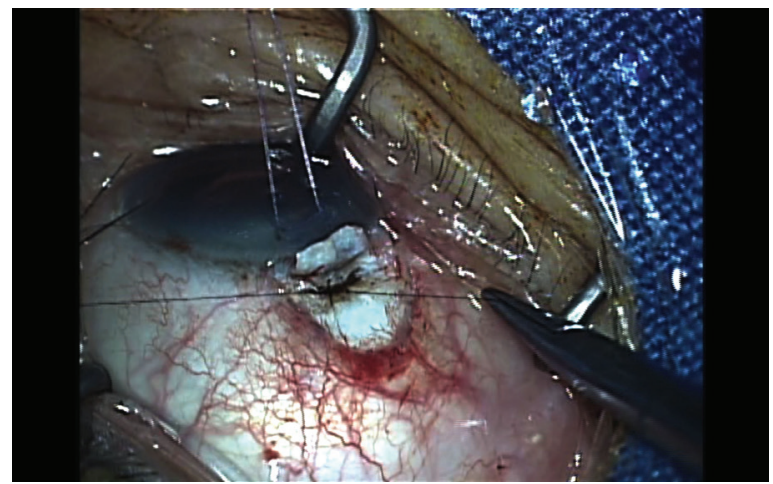

Fig. 4. Direct cyclopexy, OS. 


\section{Discussion and conclusion}

There are several aetiologies of chronic hypotony due to trauma depending on the mechanisms involved. In one study, only $6.9 \%$ of blunt trauma cases led to hypotony. ${ }^{2}$ Chronic hypotony is relatively uncommon and can be asymptomatic, and symptoms are usually due to damage to the cornea, lens, choroid, retina or optic nerve. A hyperopic shift, which was not observed in our patient, may also occur with hypotony. Choroidal effusions usually occur in hypotony due to accumulation of serous fluid in the suprachoroidal space due to the pressure gradient between the IOP and hydrostatic pressure in the choroidal blood vessels. ${ }^{3}$ Not only do choroidal effusions result from hypotony but may also lower IOP further by reducing aqueous humor production and probably through increased uveoscleral outflow as well, thus presenting a vicious cycle of hypotony. ${ }^{4} \mathrm{Hypotony}$ post-trauma can be due to ciliary shutdown, but this usually is transient; however, hypotony in our patient lasted for more than a year. When choroidal effusion is subclinical, the VA may be good and occult effusions may be diagnosed only by UBM, as seen with our patient. Hypotony maculopathy was ruled out since the macular examination was normal. Choroidal effusions eventually resolve when hypotony improves, considering that the inciting factor is managed.

Pre-operatively in our case, clinical examination and diagnostics had only revealed a shallow anterior chamber with narrow angles, with no angle recession and 360 choroidal effusions on UBM. Phacoemulsification in our case was only able to reveal the cyclodialysis cleft. The cleft or a recessed angle was probably not seen pre-operatively because of the difficulty indenting the four-mirror gonioprism due to the swollen lens and the lens-iris diaphragm pushed forward. The supine patient with the cataract already removed made it more possible to see the cleft better with the direct gonioprism.

Cyclodialysis clefts appear as a recess between the scleral spur and ciliary body. From the disrupted anatomy, aqueous may now enter the posterior chamber through the suprachoroidal space, leading to increased outflow and consequently lower IOP as well as a flat anterior chamber, resulting in the choroidal effusions. These are rare even in the setting of eye trauma; in one study, only $2 \%$ of 145 eyes studied were hypotonic and found to have an associated cyclodialysis cleft. ${ }^{2}$ It was also found that most IOPs associated with clefts are around $<7$ $\mathrm{mmHg}$ on diagnosis, with a wide range of VA among patients, whereas those with $<4 \mathrm{mmHg}$ having worse VA due to the complications from hypotony. ${ }^{5}$

There should be a high index of suspicion in cases of IOP $<5 \mathrm{mmHg}$ in cases of blunt trauma associated with hyphema, similar to our patient, or when associated with iris sphincter tears. ${ }^{6}$ Similar to choroidal effusion, these may spontaneously resolve, otherwise, may cause the complications mentioned for hypotony. Diagnostics would include gonioscopy, anterior segment OCT and UBM, although 
the latter is found to be the most sensitive to detect these clefts, especially when they are small.7 A study showed that UBM detected clefts in $100 \%$ of patients compared to only $16.7 \%$ detected by gonioscopy. ${ }^{6}$

Management of cyclodialysis clefts and subsequently chronic hypotony can range from medical to surgical, especially when the former fails. They also depend on the size of the cleft.

- Medical treatment is usually for clefts 4 clock hours or less and limited to 6 to 8 weeks with topical cycloplegics. This theoretically would allow time for fusion of the ciliary muscles to the sclera. 8 There is no consensus with the use of topical steroids to aid in the healing.

- Laser photocoagulation, diathermy or cryotherapy is the next step with unresponsive clefts and treatment modalities include argon laser photocoagulation, trans-scleral diathermy and trans-conjunctival cryotherapy or diode laser cyclophotocoagulation. ${ }^{6}$ We did not opt to do these procedures because we were contemplating to do phacoemulsification.

- Surgery is the next option if still unresponsive or with large clefts $>4$ clock hours in size. ${ }^{8}$ The various techniques are beyond the scope of this case report, but it is of note to mention that surgery was the immediate action done due to the fact that medical management had already failed and that there already was an opportunity during the cataract surgery. Despite the small size of the cleft, it had become chronic and unresponsive to the medications. Direct cyclopexy was the procedure of choice, where the ciliary body was sutured directly to the sclera through the flap created. This technique had already been established in these cases with high success rates. ${ }^{9}$

This case presented as an asymptomatic post-traumatic chronic hypotony due to subclinical choroidal effusion observed only by UBM. The aetiology was a small 2-clock-hour cyclodialysis cleft only detected by direct gonioscopy during phacoemulsification after a traumatic cataract developed. Our case also highlighted the fact that choroidal effusion could also mask a small cyclodialysis cleft. Intraoperative direct cyclopexy resulted in normalization of the IOP post-operatively.

\section{Acknowledgements}

\section{Ethics approval and consent to participate}

This observational study was approved under an expedited review by the ethics review board of the East Avenue Medical Center. 


\section{Consent for publication}

Our patient had given written consent to show photographs of his eye and printouts of his tests in this manuscript.

\section{Availability of data and materials}

The patient data used during this study are available from the corresponding author on reasonable request

\section{Competing interests}

The authors declare that they have no competing interests.

\section{Funding}

There was no source of any funding for this study.

\section{Authors' contributions}

JMSL and RSSM contributed both to the manuscript preparation. RSSM contributed to data gathering and JMSL contributed to the finalization of the article prior to submission.

\section{References}

1. Thomas M, Vajaranant TS, Aref AA. Hypotony maculopathy: clinical presentation and therapeutic methods. Ophthalmol Ther. 2015;4(2):79-88. doi:10.1007/s40123-015-0037-z.

2. Ding C, Zeng J. Clinical study on hypotony following blunt ocular trauma. Int J Ophthalmol. 2012;5(6):771. doi:10.3980/j.issn.2222-3959.2012.06.21.

3. Kahook MY, Noecker RJ. Why do choroidals form, and how do you treat them. Glaucoma Today. 2007:36-38. http://glaucomatoday.com/2007/10/GT0907_07.php/.

4. Reddy AC, Salim S. Diagnosis and management of choroidal effusions. Eyenet. 2012. https:// www.aao.org/eyenet/article/choroidal-effusions.

5. Ormerod LD, Baerveldt G, Sunalp MA, Riekhof FT. Management of the hypotonous cyclodialysis cleft. Ophthalmology. 1991;98(9):1384-1393. https://www.ncbi.nlm.nih.gov/pubmed/1945314.

6. Kiely AE, Salim S. All contributors. http://eyewiki.aao.org/Cyclodialysis_Clefts.

7. American Academy of Ophthalmology. Glaucoma 10. San Francisco, CA: American Academy of Ophthalmology; 2014.

8. Ramulu PM. Cyclodialysis cleft after trauma. Eyenet. 2004. https://www.aao.org/eyenet/article/ cyclodialysis-cleft-after-trauma.

9. Rodrigues IA, Shah B, Goyal S, Lim KS. Gonioscopically guided nonpenetrating cyclodialysis cleft repair: a novel surgical technique. J Curr Glaucoma Prac. 2017;11(1):31. https://www.ncbi.nlm. nih.gov/pmc/articles/PMC5263884/. 